\title{
Immundiagnostik bei Tumorpatienten
}

\author{
Jeden Tag entstehen Tausende Tumorzellen, die zugrunde gehen oder vom Immunsystem elimi- \\ niert werden. Doch was ist bei der einen, die sich zum Tumor entwickelt, schiefgelaufen? LTT \\ und zellulärer Immunstatus gehen dieser Frage im Labor nach und eröffnen Therapieoptionen.
}

Für die Immundiagnostik eines Krebspatienten sind zwei Fragen entscheidend:

- Wie gut funktioniert das zelluläre Immunsystem, während sich ein Tumor entwickelt? Das verrät der Lymphozytentransformationstest (LTT) als Immunfunktionstest der Lymphozyten.

- In welche Richtung ist das Immunsystem aktiviert - im Sinne zytotoxischer Abwehr oder Immunregulation und Immuntoleranz? Das verrät der zelluläre Immunstatus.

Zellulärer Immunstatus: T-Lymphozyten entscheidend Der zelluläre Immunstatus gibt einen Überblick über die Verteilung von Immunzellen. Unterteilt wird hierbei in B-Lymphozyten (CD19+), T-Lymphozyten (CD3+) und NK-Zellen (Natürliche Killerzellen - CD16/56+). Die wichtigste Rolle für eine intakte Tumorabwehr spielen die T-Lymphozyten, die in T-Helfer(CD4+) und T-Suppressorzellen (CD8+) unterschieden werden. Heute weiß man, dass beide Zellpopulationen sowohl über downregulierende als auch zytotoxische Fraktionen verfügen. Letztere sind gerade für die Tumorabwehr entscheidend.

\section{CD4+-Zellen: Immunsystem auf der Bremse?}

Die Anzahl der CD4+-Zellen liefert Hinweise zu folgenden drei Fragen:

- Sind genügend Zellen zur Abwehr oder zur Stimulation vorhanden?

- Wie hoch ist der Anteil an regulatorischen Zellen (Treg), und dementsprechend: Wie stark steht das Immunsystem auf der Bremse?

- Wie stark ist die Thymusreserve (CD31+-Zellen)? Das heißt, wie viele Zellen stehen für weitere immunstimulierbare Maßnahmen zur Verfügung?

Bei schwacher Thymusreserve kann erst einmal eine immunrestaurierende Therapie zum Beispiel mit Thymusextrakten, Vitaminen und Spurenelementen weiterhelfen.

\section{CD8+-Zellen: Aktive Tumorbekämpfung?}

Bei den CD8+-Zellen liegt das Hauptaugenmerk auf dem Verhältnis von zytotoxischen (CD28+) und regulatorischen (CD28-) Zellen. Ein hoher Anteil zytotoxischer Zellen unter- stützt den Kampf gegen Tumorzellen. Eine Erhöhung der regulatorischen CD8+-Zellen deutet hingegen auf eine „immunologische Erschöpfung“ und herunterregulierte Immunfunktion aller beteiligten Zellen hin, zum Beispiel bei chronischen Entzündungen oder Vireninfektionen. Achten Sie hier besonders darauf, dass immunstimulative Maßnahmen die zytotoxischen Funktionen und nicht den bereits hochgeregelten regulativen Anteil des Immunsystems stärken. Daher sollten Sie eine Immunstimulationstherapie bei Tumorerkrankungen nur unter Immunmonitoring durchführen und engmaschig das Verhältnis von zytotoxischen und regulativen Zellen überprüfen.

\section{Verhältnis aktivierte und präaktivierte T-Zellen}

Die präaktivierten T-Zellen (CD3+/CD25+) übernehmen die Hauptrolle in der Tumorabwehr. Denn sie sind bereit zum „Handeln" und warten förmlich auf den stimulierenden Startschuss der Therapie. Die schon terminal aktivierten T-Zellen (CD3+1 HLA DR+) sind hingegen für eine therapeutische Immunstimulation verloren, weil sie bereits auf ihr Antigen festgelegt sind und sich nicht mehr teilen.

Merke: Je höher der Anteil an aktivierten Zellen, desto genauer müssen Sie Ihre Therapie abwägen, um eine Überstimulation zu vermeiden.

\section{NK-Zellen: Blutwert wenig aussagekräftig}

Die quantitative Messung der NK-Zellen spielt eine untergeordnete Rolle. Denn diese sind zur Tumorabwehr häufig ins Gewebe ausgewandert und nur deshalb zahlenmäßig im Blut vermindert. Entscheidend ist hingegen die Aussage über ihre Funktionalität. Diese kann mithilfe des NK-Zell-Zytotoxizitätstests getroffen werden. Hierbei wird in vitro gemessen, wie viele Tumorzellen von den NK-Zellen in einer bestimmten Zeit in die Apoptose getrieben werden und wie stark sich die NK-Zellfunktion durch eine Immunstimulation verbessert.

\section{LTT ermittelt Immunfunktion und optimale Therapie}

Der Lymphozytentransformationstest LTT dient als Funktionstest der Einschätzung der Immunfunktion und zum Ermitteln der individuell geeignetsten Immunstimulanzien. Dafür werden 
im Labor aus dem Patientenblut Lymphozyten gewonnen und in einer Zellkultur mit verschiedenen, größtenteils bekannten Antigenen (zum Beispiel Tetanus, Candida, Zytomegalievirus) konfrontiert. Weil nicht alle Patienten zu allen Antigenen Kontakt hatten, werden mehrere getestet. Eine herausstechende Immunantwort auf einen Erreger weist andererseits auf eine verstärkte aktuelle Auseinandersetzung hin.

Nach einigen Tagen wird die Immunantwort anhand der Lymphozytenvermehrung in der jeweiligen Antigengruppe bestimmt. Daraus errechnet sich der Mittlere Funktionsindex (MFI) als Gradmesser der zellulären Immunfunktion. Er sollte beim Gesunden über 15 liegen. Messen wir die Immunfunktion bei einem Krebspatienten, kommt es bei der Interpretation auf den Krankheits- bzw. Therapieverlauf an: Bei frisch diagnostiziertem, progredientem Tumorverlauf erwartet man eine starke Abwehr (MFI >20), postoperativ und tumorfrei eine „normale“ Immunfunktion (MFI >15), nach einer Chemotherapie eine deutliche Absenkung.

Parallel wird die Reaktion auf verschiedene Immunstimulanzien wie Thymusextrakt, Iscador ${ }^{\circledR} \mathrm{M}$, Iscador ${ }^{\circledR} \mathrm{P}$ (jeweils Fa. Weleda), Shitake oder Helixor ${ }^{\circledR}$ A (Fa. Helixor) getestet und somit die optimale Therapie ermittelt.

\section{Merke: Der beste Zeitpunkt zur Erstuntersuchung des Immunsystems bei einer Tumorerkrankung ist direkt nach der Diagnose, ohne Einfluss von Medikamenten oder anderen therapeutischen Maßnahmen.}

Bei niedrigem MFI $(<10)$, also einer reduzierten Immunfunktion, hat das körpereigene Abwehrsystem nicht die erforderliche Immunkompetenz, um den Tumor zu bekämpfen oder am Wachstum zu hindern. In den meisten Fällen sehen wir parallel im zellulären Immunstatus ein ungünstiges Verhältnis von re- gulativen und zytotoxischen Zellen. Die richtige Therapie besteht dann in einer gezielten und individuellen Immunstimulation nach dem Ergebnis des LTT-Stimulationstest und gegebenenfalls einer Herdsanierung.

Bei einem MFI um 15 haben wir zwar eine gute Immunfunktion, aber keine erkennbare Aktivierung durch ein vorhandenes Tumorgeschehen. Um diesen Escape-Mechanismus (Strategie, sich der Immunabwehr zu entziehen) zu durchbrechen, stehen der Naturheilkunde einige Möglichkeiten zur Verfügung. Dazu zählen unter anderem Ganzkörperhyperthermie, miasmatische Homöopathie oder Enzymtherapie, jeweils in Kombination mit geeigneten Immunstimulanzien.

Ein hoher MFI (> 20) spricht für eine aktive Immunantwort gegen das Tumorgeschehen. Eine therapeutische Immunstimulation sollte hier nur unter Laborkontrolle (Verhältnis zytotoxischer versus regulativer CD4- und CD8-Zellen) erfolgen, um eine Überstimulation zu vermeiden.

\section{Kontrolle: Therapie erfolgreich und weiterhin angezeigt?}

Bei der Wiederholung der Analysen acht bis zehn Wochen nach Beginn der immunstimulierenden Therapie wird mit dem LTT überprüft, ob der MFI angestiegen ist (als Maßstab für eine erfolgreiche Therapie) und das ausgewählte Präparat noch immer optimal anspricht. Auch ein Missverhältnis der regulativen und zytotoxischen Zellen sollte sich jetzt bereits verbessert haben.

HP Enrico Thiele, Stuttgart, Dr. med. Volker von Baehr, Berlin

Dieser Artikel ist online zu finden:

http://dx.doi.org/10.1055/s-0043-109394
Zelluläre Immunfunktion

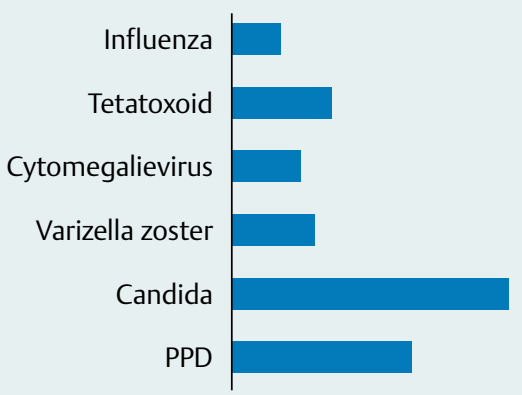

Mittlerer Funktionsindex: $\quad 9,3$

Aus dem Mittelwert der 6 antigenstimulierten Indizes errechnet sich der mittlere Funktionsindex (siehe Feld darunter), der besser als die Einzelparameter zur Beurteilung und Verlaufskontrolle der Immunfunktion geeignet ist.

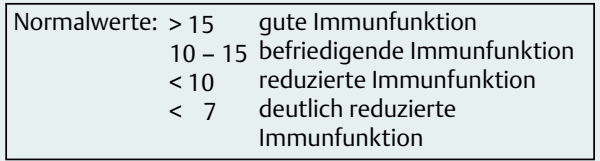

3,5

7,3

5,1

5,8

20,9

13,1

Reaktivität gegenüber Immunstimulatoren

Thymusextrakt

Iscador ${ }^{\circledR} \mathrm{M}$

Iscador ${ }^{\circledR} \mathrm{P}$

Shitake

Utilin ${ }^{\circledR}$

Helixor ${ }^{\circledR} \mathrm{A}$
1,0

6,1

1,0

1,0

1,0

5,3

\begin{tabular}{|l|c|}
\hline Leerwert (Negativkontrolle) & 907 (Normalwert < 4000 cpm) \\
\hline Mitogenkontrolle (PWM) & 22832 (Normalwert > 20000 cpm) \\
\hline
\end{tabular}

Abb. 1 Beispiel eines niedrigen Mittleren Immunfunktionsindex (MFI). Hier wäre eine immunstimulierende Therapie indiziert. Zudem zeigt der Test, dass die Verwendung der getesteten Mistelpräparate Iscador ${ }^{\circledR} \mathrm{M}$ (Fa. Iscador) oder Helixor ${ }^{\circledR} \mathrm{A}$ (Fa. Helixor) erfolgversprechend ist. Foto: ๑ Volker von Baehr 